\title{
14-3-3 protein dependent modulation of ubiquitin ligase Nedd4-2
}

\author{
P. Pohl ${ }^{1,2}$, T. Obsil ${ }^{1,3}$ a V. Obsilova ${ }^{1}$ \\ ${ }^{1}$ Dept. of Structural Biology of Signalling Proteins, Division BIOCEV, Institute of Physiology of the Czech Academy of Sciences, \\ Vestec, Czech Republic \\ ${ }^{2}$ Second Faculty of Medicine, Charles University in Prague, Czech Republic \\ ${ }^{3}$ Dept. of Physical and Macromolecular Chemistry, Faculty of Science, Charles University in Prague, Czech Republic \\ pavel.pohl@fgu.cas.cz
}

Neural precursor cell expressed developmentally down-regulated 4 ligase (Nedd4-2) is an E3 ubiquitin ligase that targets proteins for ubiquitination and endocytosis, thereby regulating numerous ion channels, membrane receptors and tumor suppressors. In turn, Nedd4-2 activity is regulated by autoinhibition, calcium binding, oxidative stress, substrate binding (through its WW domains), phosphorylation and 14-3-3 protein binding [1-3]. However, the structural basis of 14-3-3-mediated Nedd4-2 regulation remains poorly understood.

Here, we combined several techniques of integrative structural biology to characterize Nedd4-2 and its complex with 14-3-3. The results from our binding affinity and crystallographic analyses demonstrate that phosphorylated $\mathrm{Ser}^{342}$ and $\mathrm{Ser}^{448}$ are the key residues that facilitate 14-3-3 protein binding to Nedd4-2 and that $\operatorname{Ser}^{448}$ is the dominant site. Moreover, 14-3-3 protein binding induces a structural rearrangement of Nedd4-2 by inhibiting interactions between its structured domains, including the N- and C-lobes of the catalytic HECT domain. Overall, our findings provide the first structural glimpse into the 14-3-3-mediated Nedd4-2 regulation and highlight the potential of the Nedd4-2:14-3-3 complex as a pharmacological target for Nedd4-2-associated diseases such as hypertension, epilepsy, kidney disease and cancer.

[1] P. Goel, J. A. Manning, and S. Kumar, Gene, 557, no. 1, pp. 1-10, Feb. 2015.

[2] H. He, C. Huang, Z. Chen, H. Huang, X. Wang and J. Chen, Biomed Pharmacother, 125, no. 1, pp. 109983, Feb. 2020.

[3] J. A. Manning and S. Kumar, Trends Biochem. Sci., 43, no. 8, pp. 635-647, Aug. 2018.

\section{Keywords: SAXS, chemical crosslinking, crystallography, 14-3-3, Nedd4-2, ubiquitination, phosphorylation}

This study was supported by the Czech Science Foundation (Project 20-00058S), the Czech Academy of Sciences (Research Projects RVO: 67985823 of the Institute of Physiology) and by Grant Agency of Charles University (Project 740119). 